\title{
The Frequency of Physiologic Monitor Alarms in a Children's Hospital
}

Amanda C. Schondelmeyer, MD, MSc ${ }^{1,2 \star}$, Christopher P. Bonafide, MD, MSCE ${ }^{3}$, Veena V. Goel, MD4, Nancy Blake, PhD, RN5, Maria Cvach, DNP, RN , Heidi Sucharew, $\mathrm{PhD}^{7}$, Jeffrey M. Simmons, MD, MSc ${ }^{1,2}$, Patrick W. Brady, MD, MSc ${ }^{1,2}$

${ }^{1}$ Division of Hospital Medicine, Department of Pediatrics, Cincinnati Children's Hospital Medical Center, Cincinnati, Ohio; 2James M. Anderson Center for Health Systems Excellence, Cincinnati Children's Hospital Medical Center, Cincinnati, Ohio; 'Division of General Pediatrics, The Children's Hospital of Philadelphia, Philadelphia, Pennsylvania; ' Division of Hospital Medicine, Lucile Packard Children's Hospital Stanford, Palo Alto, California; ${ }^{5}$ Division of Critical Care, Children's Hospital Los Angeles, Los Angeles, California; ${ }^{6}$ Department of Nursing, Johns Hopkins Hospital, Baltimore, Maryland; 'Division of Biostatistics and Epidemiology, Cincinnati Children's Hospital Medical Center, Cincinnati, Ohio.

Physiologic monitor alarms are an inescapable part of the soundtrack for hospitals. Data from primarily adult hospitals have shown that alarms occur at high rates, and most alarms are not actionable. ${ }^{1}$ Small studies have suggested that high alarm rates can lead to alarm fatigue. ${ }^{2,3}$ To prioritize alarm types to target in future intervention studies, in this study we aimed to investigate the alarm rates on all inpatient units and the most common causes of alarms at a children's hospital.

\section{METHODS}

This was a cross-sectional study of audible physiologic monitor alarms at Cincinnati Children's Hospital Medical Center (CCHMC) over 7 consecutive days during August 2014. CCHMC is a 522-bed freestanding children's hospital. Inpatient beds are equipped with GE Healthcare (Little Chalfont, United Kingdom) bedside monitors (models Dash 3000, 4000, and 5000, and Solar 8000). Age-specific vital sign parameters were employed for monitors on all units.

We obtained date, time, and type of alarm from bedside physiologic monitors using Connexall middleware (GlobeStar Systems, Toronto, Ontario, Canada).

We determined unit census using the electronic health records for the time period concurrent with the alarm data collection. Given previously described variation in hospital census over the day, ${ }^{4}$ we used 4 daily census measurements (6:00 AM, 12:00 PM, 6:00 PM, and 11:00 PM) rather than 1 single measurement to more accurately reflect the hospital census.

The CCHMC Institutional Review Board determined this work to be not human subjects research.

*Address for correspondence and reprint requests: Amanda $\mathrm{C}$. Schondelmeyer, MD, Cincinnati Children's Hospital Medical Center, 3333 Burnet Avenue ML 9016, Cincinnati, OH 45229; Telephone: 513-803-

9158; Fax: 513-803-9224; E-mail: amanda.schondelmeyer@cchmc.org

Received: April 1, 2016; Accepted: April 28, 2016

2016 Society of Hospital Medicine DOI 10.1002/jhm.2612

Published online in Wiley Online Library (Wileyonlinelibrary.com).

\section{Statistical Analysis}

For each unit and each census time interval, we generated a rate based on the number of occupied beds (alarms per patient-day) resulting in a total of 28 rates ( 4 census measurement periods per/day $\times 7$ days) for each unit over the study period. We used descriptive statistics to summarize alarms per patient-day by unit. Analysis of variance was used to compare alarm rates between units. For significant main effects, we used Tukey's multiple comparisons tests for all pairwise comparisons to control the type I experiment-wise error rate. Alarms were then classified by alarm cause (eg, high heart rate). We summarized the cause for all alarms using counts and percentages.

\section{RESULTS}

There were a total of 220,813 audible alarms over 1 week. Median alarm rate per patient-day by unit ranged from 30.4 to 228.5; the highest alarm rates occurred in the cardiac intensive care unit, with a median of 228.5 (interquartile range [IQR], 193-275) followed by the pediatric intensive care unit (172.4; IQR, 141-188) (Figure 1). The average alarm rate was significantly different among the units $(P<0.01)$.

Technical alarms (eg, alarms for artifact, lead failure), comprised $33 \%$ of the total number of alarms. The remaining $67 \%$ of alarms were for clinical conditions, the most common of which was low oxygen saturation (30\% of clinical alarms) (Figure 2).

\section{DISCUSSION}

We described alarm rates and causes over multiple units at a large children's hospital. To our knowledge, this is the first description of alarm rates across multiple pediatric inpatient units. Alarm counts were high even for the general units, indicating that a nurse taking care of 4 monitored patients would need to process a physiologic monitor alarm every 4 minutes on average, in addition to other sources of alarms such as infusion pumps.

Alarm rates were highest in the intensive care unit areas, which may be attributable to both higher rates of monitoring and sicker patients. Importantly, however, alarms were quite high and variable on the acute 
Unit

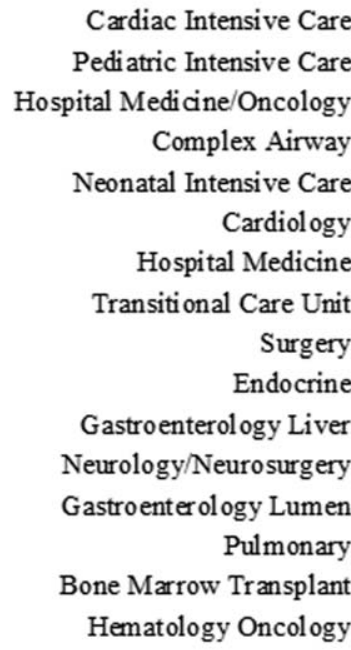

Average

(standard deviation)

$233.5(70.5)$

$172.9(46.9)$

$149.6(64)$

$123.4(113.2)$

$121.2(21.1)$

$103.8(62.5)$

$93.2(61.2)$

$91.7(32.5)$

$75.7(46)$

$75(66.2)$

$73.9(20.1)$

$67.3(20.1)$

$61.9(32)$

$54.5(30.8)$

$45.4(12.1)$

$32.3(13.5)$
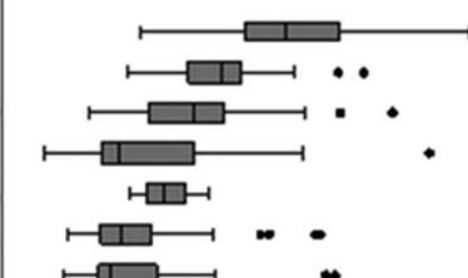

$\mapsto \square$ -
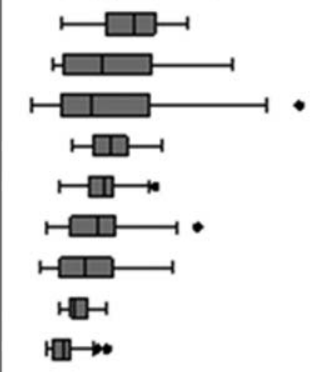

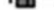

Alarms per Patient-Day

FIG. 1. Alarm rates by unit over 28 study observation periods.

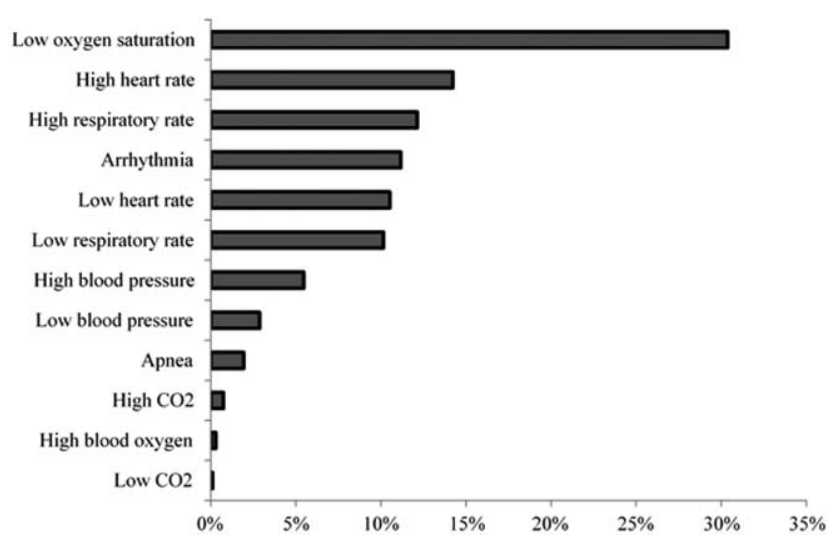

FIG. 2. Causes of clinical alarms as a percentage of all clinical alarms. Technical alarms, not included in this figure, comprised $33 \%$ of all alarms.

care units. This suggests that factors other than patient acuity may have substantial influence on alarm rates.

Technical alarms, alarms that do not indicate a change in patient condition, accounted for the largest percentage of alarms during the study period. This is consistent with prior literature that has suggested that regular electrode replacement, which decreases technical alarms, can be effective in reducing alarm rates. ${ }^{5,6}$ The most common vital sign change to cause alarms was low oxygen saturation, followed by elevated heart rate and elevated respiratory rate. Whereas in most healthy patients, certain low oxygen levels would prompt initiation of supplemental oxygen, there are many conditions in which elevated heart rate and respiratory rate may not require titration of any particular therapy. These may be potential intervention targets for hospitals trying to improve alarm rates.

\section{Limitations}

There are several limitations to our study. First, our results are not necessarily generalizable to other types of hospitals or those utilizing monitors from other vendors. Second, we were unable to include other sources of alarms such as infusion pumps and ventilators. However, given the high alarm rates from physiologic monitors alone, these data add urgency to the need for further investigation in the pediatric setting.

\section{CONCLUSION}

Alarm rates at a single children's hospital varied depending on the unit. Strategies targeted at reducing technical alarms and reducing nonactionable clinical alarms for low oxygen saturation, high heart rate, and high respiratory rate may offer the greatest opportunity to reduce alarm rates.

\section{Acknowledgements}

The authors acknowledge Melinda Egan for her assistance in obtaining data for this study and Ting $\mathrm{Sa}$ for her assistance with data management.

Disclosures: Dr. Bonafide is supported by the National Heart, Lung, and Blood Institute of the National Institutes of Health under award number K23HL116427. Dr. Bonafide also holds a Young Investigator Award grant from the Academic Pediatric Association evaluating the impact of a data-driven monitor alarm reduction strategy implemented in safety huddles. Dr. Brady is supported by the Agency for Healthcare Research and Quality under award number K08HS23827. The content is solely the responsibility of the authors and does not necessarily represent the official views of the National Institutes of Health or the Agency for Healthcare Research and Quality. This study was funded by the Arnold W. Strauss Fellow Grant, Cincinnati Children's Hospital Medical Center. The authors have no conflicts of interest to disclose.

\section{References}

1. Paine CW, Goel VV, Ely E, et al. Systematic review of physiologic monitor alarm characteristics and pragmatic interventions to reduce alarm frequency. J Hosp Med. 2016;11(2):136-144. 
2. Bonafide CP, Lin R, Zander M, et al. Association between exposure to nonactionable physiologic monitor alarms and response time in a children's hospital. J Hosp Med. 2015;10(6):345-351.

3. Voepel-Lewis T, Parker ML, Burke CN, et al. Pulse oximetry desaturation alarms on a general postoperative adult unit: a prospective observational study of nurse response time. Int I Nurs Stud. 2013; 50(10):1351-1358.

4. Fieldston E, Ragavan M, Jayaraman B, Metlay J, Pati S. Traditional measures of hospital utilization may not accurately reflect dynamic patient demand: findings from a children's hospital. Hosp Pediatr. 2012;2(1):10-18.

5. Dandoy CE, Davies SM, Flesch L, et al. A team-based approach to reducing cardiac monitor alarms. Pediatrics. 2014;134(6):e1686e1694.

6. Cvach MM, Biggs M, Rothwell KJ, Charles-Hudson C. Daily electrode change and effect on cardiac monitor alarms: an evidence-based practice approach. J Nurs Care Qual. 2013;28(3): 265-271. 